\title{
DETECTION OF INDIVIDUAL NORMAL MODES \\ OF OSCILLATION OF THE SUN IN THE PERIOD RANGE \\ FROM 2 HR TO 10 MIN \\ IN SOLAR DIAMETER STUDIES*
}

\author{
RANDALL J. BOS and HENRY A. HILL \\ Department of Physics and Arizona Research Laboratories, University of Arizona, Tucson, \\ AZ 85721, U.S.A.
}

\begin{abstract}
New observations of solar oscillations are reported. Power density spectra derived from these observations reveal narrow-band oscillations that are spatially global, have spatial symmetry properties that are either symmetric or antisymmetric for reflection about the center of the solar disk and also about the solar equator, and have coherence times $\gtrsim 41$ days. Large-scale differential refraction effects have been reduced by a factor of $10^{5}$ over that found in previous solar diameter studies by the design of the experiment; thus, these effects are eliminated as a possible source of the oscillations. A discussion is presented of this reduction as well as other features of the observing and analysis program. It is concluded that the probability is very high that individual normal modes of oscillation of the Sun have been detected in the period range from $2 \mathrm{hr}$ to $10 \mathrm{~min}$.
\end{abstract}

\section{Introduction}

Evidence of solar oscillations has been obtained from meaurements of solar diameters made in 1973, 1975, and 1978 (Brown et al., 1978; Hill and Caudell, 1979; Caudell and Hill, 1980; Caudell et al., 1980). This paper reports the preliminary analysis of observations obtained in 1979.

The 1979 observations are yielding much more information than the previous three sets of observations because of changes in the solar detector which lead to more detailed spatial information and better signal-to-noise ratio and because of a longer time span for the observations. The improved spatial information permits identification of certain spatial symmetry properties of the eigenfunctions for reflections about the center of the solar disk and about the solar equator. These properties are invaluable in mode classification. They may be equally useful in evaluating the viability of such solar models as the oblique magnetic rotator model proposed by Dicke (1978) and recently brought forward by Isaak (1982). From an observational point of view, the additional spatial information makes it possible to significantly reduce the effects of differential refraction in the Earth's atmosphere, because their spatial characteristics can be readily distinguished from those of solar oscillations. An improved signal-to-noise ratio is obtained because of the simultaneous observation of three solar diameters instead of one as in previous work. The time span for the 1979 data set is 41 days, leading to a

\footnotetext{
* Proceedings of the 66th IAU Colloquium: Problems in Solar and Stellar Oscillations, held at the Crimean
} Astrophysical Observatory, U.S.S.R., 1-5 September, 1981. 
frequency resolution of $0.28 \mu \mathrm{Hz}$. For oscillations which are stable for periods of time $\gtrsim 41$ days, this increased time span also contributes to an improved signal-to-noise ratio.

Power density spectra derived from this data set spanning 41 days offer a frequency resolution of $0.28 \mu \mathrm{Hz}$. However, with such analyses, one is often left with the difficult task of separating the signal from the noise. One way to address this is to subdivide the data set into two or more subsets and compare the power density spectra of these smaller sets. This technique, used in some variation in all of the previous analyses of solar diameter measurements, was successfully employed in the analysis of the 1979 data. However, the variation used in this latter analysis allowed for the identification of statistically significant oscillatory signals while maintaining frequency resolution which is adequate to distinguish individual modes of oscillation.

It has been recognized for some time that the oscillatory signals obtained in solar diameter measurements at SCLERA* are the direct manifestation of fluctuations in the solar limb darkening function. In the previous work, the data were analyzed in various ways to demonstrate this property. In the analysis of the 1979 observations, full advantage of this property is taken by using it as a means to significantly reduce atmospheric differential refraction effects and to separate the signal from the noise arising from a broad class of noise sources. As a consequence, individual eigenstates of the Sun have been clearly seen for the first time in the frequency range $125 \mu \mathrm{Hz}$ to $1.5 \mathrm{mHz}$ (periods between $\approx 2 \mathrm{hr}$ and $10 \mathrm{~min}$ ). This statement, based on confidence levels typically $>99.9 \%$, represents significant progress in the development of the field of helioseismology.

\section{Observations}

The solar diameter observations that are used here to identify individual eigenstates of the Sun were obtained in June and July of 1979 at SCLERA. These observations are of the same general class as those made at SCLERA starting in 1972 with Clayton (1973) and Patz (1975), and followed by Hill and Stebbins (1975), Brown et al. (1978), and Caudell et al. (1980). These preceding works defined positions on diametrically opposite limbs and then observed the change in the separation of these positions as a function of time. The positions on the limbs are determined by the Finite Fourier Transform Definition (FFTD) (see Hill et al., 1975; Hill, 1978) and the relative changes in the edge separation recorded interferometrically. A detailed description of the data-taking procedure is found in these earlier works and a description of the facility is provided by Oleson et al. (1974).

However, there are two important differences between the earlier work and that done in 1979. The most recent work utilized information on six limb positions rather than on the separation of only two diametrically opposite edges. These six limb positions form

* SCLERA is an acronym for the Santa Catalina Laboratory for Experimental Relativity by Astrometry, a facility jointly operated by the University of Arizona and Wesleyan University. 
three diametrically opposite pairs with the center diameter coinciding with the equator and the other two being rotated $\frac{1}{8}$ of a radian either side of the equator. The slit configuration is shown in Figure 1.

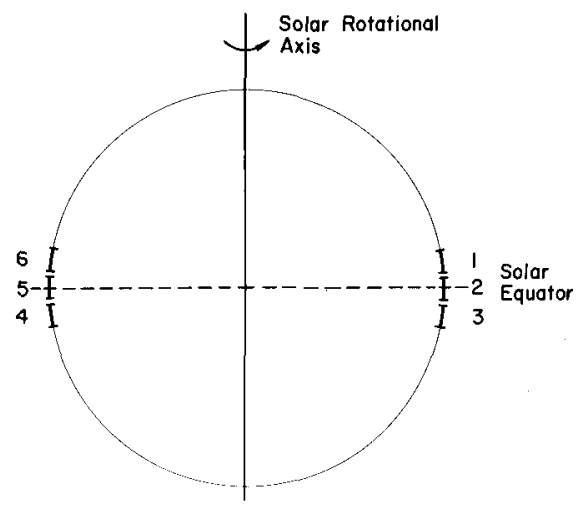

Fig. 1. Slit configuration relative to the solar disk.

The second important difference is that at each of the six limb positions, two different edges, $r_{1}(\theta, \phi)$ and $r_{2}(\theta, \phi)$, were defined by applying the FFTD first with a scan amplitude $a_{1}$ of $6.8 \mathrm{arc} \mathrm{sec}$ and then with a scan amplitude $a_{2}$ of $27.2 \mathrm{arcsec}$. The angles $\theta$ and $\phi$ are the spherical coordinates of the slit position on the limb (see Figure 2). The separation of these two edges is typically $3.5 \mathrm{arcsec}$ (see Hill et al., 1975). It is the separation of these two edges, $S_{i}$ with $i=1-6$, that is recorded for each of the six limbs and combinations of the six sets of data that are subsequently Fourier analyzed for evidence of solar oscillations. The $S_{i}$ are defined such that a positive value of $S_{i}$ indicates net displacement in the positive radial direction.

It has been well-documented that the oscillations are detected primarily through changes in the radiation intensity, i.e., perturbations in temperature, with spatial frequencies on the order of one $(\operatorname{arc~sec})^{-1}$ near the limb (Hill and Caudell, 1979; Knapp et al., 1980). Because of this, the sensitivity of the $S_{i}$ to oscillations is quite similar to that obtained in diameter measurements, $r_{1}(\theta, \phi)+r_{1}(\pi-\theta, \pi+\phi)$. However, the

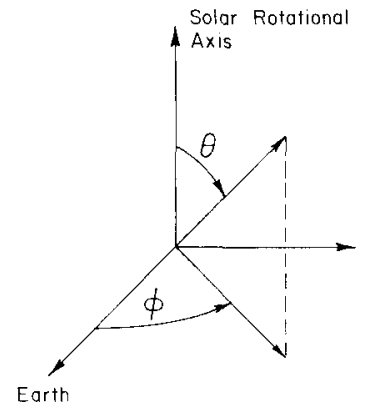

Fig. 2. Spherical coordinate system defined relative to the solar rotational axis. 
sensitivity of the $S_{i}$ relative to that of the diameter measurements is significantly reduced for all sources of noise which change the scale in the telescope, such as differential refraction in the Earth's atmosphere. This clearly discriminates against certain problems that may otherwise be presented by the Earth's atmosphere. Section 8 evaluates this important point in more detail.

Power density spectra for various combinations of the signals, $S_{i}$, were calculated. In this, the mean of $S_{i}$ for each day was removed and a $10 \%$ cosine bell applied to the residual, $S_{i}-\bar{S}_{i}$, for each day. Information from these various combinations is used in the following sections to ascertain various properties of the data and oscillations. An example of the power density spectrum is shown in Figure 3 for a $30 \mu \mathrm{Hz}$ frequency range near $450 \mu \mathrm{Hz}$. This power density function is for the combination of signals:

$$
\left(\sum_{i=1}^{6} S_{i}\right) / 6 .
$$

Because of the sign convention on the individual $S_{i}$, this combination of $S_{i}$ is sensitive to eigenfunctions that are symmetric about the equator of the Sun and symmetric for reflection through the center of the solar disk $(\theta \rightarrow \pi-\theta ; \phi \rightarrow \pi+\phi)$.

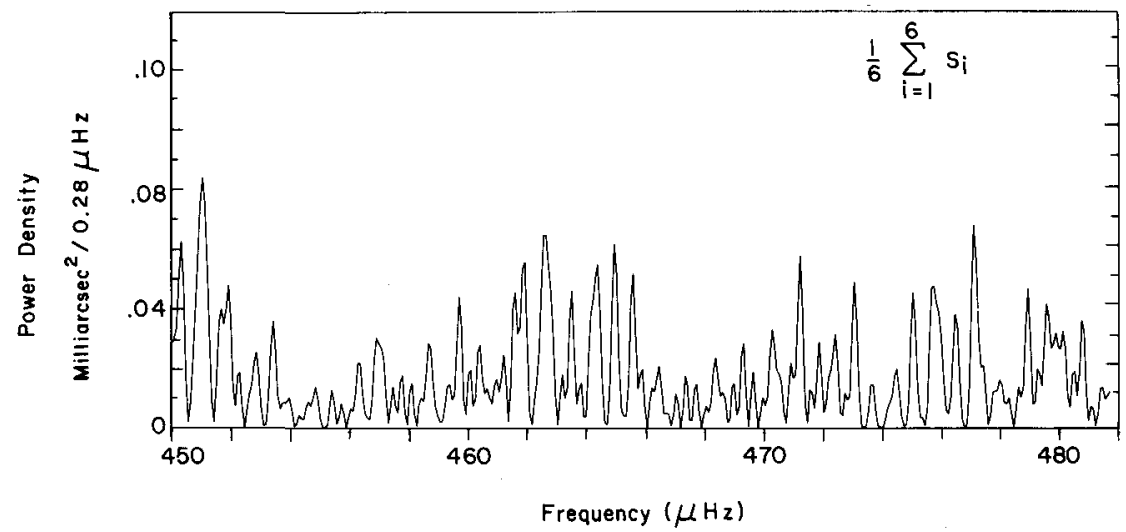

Fig. 3. Power density spectrum of the slit combination $\left(S_{1}+S_{2}+S_{3}+S_{4}+S_{5}+S_{6}\right)$ for the frequency range 450 to $480 \mu \mathrm{Hz}$.

\section{Fractional Power Due to Temporally and Spatially Noncoherent Signals}

Noncoherent signal in the power density spectrum can be classified as such by either of two criteria: signals having spatial coherence lengths which are less than a solar diameter or those with temporal coherence times less than 2 days. In principle, signals can exist that meet only one of these criteria, but signals arising from global oscillations should not meet either. The 1979 observations can be analyzed using both criteria.

\section{A. SPatially NONCOHERENT Signals}

Six independent signals, $S_{i}$ for $i=1-6$, are acquired from the six separate limb positions. 
From a given combination of the $S_{i}$, the quantity of interest is the fraction of power density which is due to spatially coherent signals. The following model is adopted for defining a measure of coherence: a spatially coherent signal will have a coherence length $>$ a solar diameter, while a noncoherent signal will have a coherence length $\lesssim 100$ arc sec; in addition, the coherent signals are symmetric or antisymmetric for reflections about the disk center and also for reflections about the equator. Should the measure of coherence turn out to be less than $100 \%$ by a statistically significant amount, then the assumptions in the above model may not be valid although a certain degree of coherence may be established. If, on the other hand, the measure of coherence approaches $100 \%$, then this result is also a measure of the validity of the above model. The combination of $S_{i}$ that has been examined for spatial coherence is

$$
\left(\sum_{i} S_{i}\right) / 6,
$$

the combination used for the power density reported in Figure 3. For this combination, only the spatially coherent signals that are symmetric for reflections both about the disk center and the equator survive. For this combination of $S_{i}$ and using the model outlined in the preceding paragraph, the measure of coherence, $\gamma$, can be written as

$$
\gamma=\frac{3}{2}\left(1-\frac{2 y}{x}\right),
$$

where

$$
\begin{aligned}
x= & \frac{1}{6}\left|F\left(\sum_{i} S_{i}\right)\right|^{2}, \\
y= & \frac{1}{6} \sum_{i=1}^{6}\left|F\left(S_{i}\right)\right|^{2}-\left|F\left(\frac{S_{1}+S_{2}+S_{3}-S_{4}-S_{5}-S_{6}}{6}\right)\right|^{2}- \\
& -\frac{2}{3}\left|F\left(\frac{S_{1}+S_{4}-S_{3}-S_{6}}{4}\right)\right|^{2}-\frac{2}{3}\left|F\left(\frac{S_{1}-S_{4}-S_{3}+S_{6}}{4}\right)\right|^{2}
\end{aligned}
$$

and $F(f)$ is the Fourier transform of function $f$. This coherence function is normalized such that $\gamma=1$ for a $100 \%$-coherent signal and $\gamma=0$ when there is no spatially coherent signal present. Thus, any statistically significant departure of $\gamma$ from zero is a direct measure of the component in the $S_{i}$ that is spatially coherent in the combination $\sum S_{i}$.

Using the observational data in the frequency range from $400 \mu \mathrm{Hz}$ to $450 \mu \mathrm{Hz}$, the calculated coherence function $\gamma$ implies that $98 \% \pm 5 \%$ of the power density is spatially coherent and that $2 \% \pm 5 \%$ is spatially noncoherent. The power density of the spatially noncoherent type, $P_{s, n c}$, is thus

$$
P_{s, n c}=(0.5 \pm 1.3) \times 10^{-3}(\text { milli arc sec })^{2} / 0.28 \mu \mathrm{Hz} .
$$


As previously noted, the fact that $\gamma$ is found to be quite close to one serves as a check on the validity of the assumptions that went into the derivation of Equation (2). The validity of these assumptions is also supported by the results from Section 5 on symmetry properties.

\section{B. TEMPORALLY NONCOHERENT SIGNALS}

In a procedure which is an extension of that considered by Groth (1975), temporal coherence was examined by numbering the days of the data set sequentially from one to 18 and then dividing the days into even-numbered and odd-numbered sets. The frequency resolution for power density spectra from these two data sets is essentially the same as that for the total 18-day set spanning 41 days since the mean length of both data sets is $\approx 38$ days. Any variation in power density in the peaks from the even- to odd-numbered spectra must result from signals with coherence times $<2$ days, the mean spacing of the 18 days of data. If signals from any eigenstates are present, they will very likely be resolved and cannot give rise to any interference effects to complicate the interpretation.

The decision to set the boundary distinguishing coherent and noncoherent signals at approximately 2 days was dictated in part by signal-to-noise considerations in the design of the study. It was also recognized that a 2-day limit is sufficiently long to discriminate effectively against most noise sources such as the Earth's atmosphere and the observing instrument.

A comparison of the even-and odd-numbered power density spectra was made for the frequency range 400 to $500 \mu \mathrm{Hz}$. From the average of the square of the observed scatter in the peak power densities, $98.9 \%$ of the power density was found to be temporally coherent and $1.1 \%$ temporally noncoherent. The power density of the temporally noncoherent signal, $P_{t, n c}$, is thus

$$
P_{t, n c}=4.1 \times 10^{-4}(\text { milli } \operatorname{arc~sec})^{2} / 0.28 \mu \mathrm{Hz} .
$$

The high proportion of temporally coherent vs noncoherent power density should enhance the statistical significance of any results that may be drawn from the analysis of these observations.

The determination of the temporally noncoherent power density level is approximately ten times more accurate than that for the spatially noncoherent power density level. It is interesting to note that the two independently determined power levels are consistent with the hypothesis that temporal and spatial coherence must both be associated with an oscillation. This conclusion is further supported by the observed symmetry properties discussed in Section 5.

\section{Phase Coherence Time $>41$ Days}

After classifying the observed power density as coherent or noncoherent, the next question to be addressed concerns the actual length of the phase coherence. The 
question arises in part because the future of the field of helioseismology depends on the ability to study the spatial properties of individual solar eigenstates and to measure very accurately the eigenfrequencies themselves (Gough, 1978). Both of these can only be achieved if the coherence times are $\gg$ days.

An initial approach to this problem might be to extend the technique used to establish the $1.1 \%-98.9 \%$ distribution of noncoherent to coherent power from a limiting coherence time of, for example, 2 days to 4 days or more. However, this procedure brings about a rapid decrease in the signal-to-noise ratio, making some other analysis possibly more attractive.

A concept of correlation time (Stratonovich, 1963) that gives some idea of the size of the time interval over which correlation extends between values of a process is defined by the relation

$$
\tau_{\text {cor }}=\frac{1}{K(0)} \int_{0}^{\infty}|K(\tau)| \mathrm{d} \tau,
$$

where

$$
K(\tau)=\int_{v_{1}}^{v_{2}} P e^{-i 2 \pi v \tau} \mathrm{d} v
$$

and $P$ is the power density spectrum. This correlation time is appropriate for the oscillatory signals with frequency between $v_{2}$ and $v_{1}$ as long as $\tau_{\operatorname{cor}} \gg 1 /\left(v_{2}-v_{1}\right)$.

The $\tau_{\text {cor }}$ was calculated for the power density of $\sum S_{i} / 6$ over the frequency range 450 to $480 \mu \mathrm{Hz}$ and compared to that expected for oscillations with autocorrelation functions

$$
K(\tau)=K(0) e^{-\beta|\tau|} \cos \omega_{0} \tau .
$$

The results of this analysis yield

$$
\beta^{-1}=51 \text { days } \text {. }
$$

Therefore, the coherence time of these oscillations must be $>41$ days.

\section{Spatial Symmetries of Observed Eigenfunctions}

The identification of spatial symmetries in the observed eigenfunctions can be valuable for several reasons. Should the phase and amplitude of an oscillation at one solar limb have a significant and well-defined relation to that of an oscillation at a diametrically opposite limb, the global character of the oscillation is demonstrated. The classification of the symmetry properties also helps to identify any preferred direction within the Sun, a preferred direction which might arise, for example, as a result of a Sun that is not spherically symmetrical as seen by the oscillations. In addition, symmetry properties might provide a means for ascertaining whether the oscillations originate in the Sun or the Earth's atmosphere. For example, symmetries that clearly reflect an axis on the Sun 
would be quite difficult to ascribe to effects arising in the Earth's atmosphere while, on the other hand, symmetries that reflect the local elevation and azimuth system of the observatory would be hard to interpret in terms of a solar phenomenon.

The observations reported here were made in such a way so as to detect changes in the solar limb darkening function at six different places on the solar perimeter. These locations are shown in Figure 1, with the details of the geometry given in Section 2 . The more interesting combinations of the $S_{i}$ are

$$
\begin{aligned}
& \left(S_{1}+S_{2}+S_{3}\right) \pm\left(S_{4}+S_{5}+S_{6}\right), \\
& \left(S_{1}+S_{4}\right) \pm\left(S_{3}+S_{6}\right), \\
& \left(S_{1}-S_{4}\right) \pm\left(S_{3}-S_{6}\right) .
\end{aligned}
$$

Analysis of these combinations shows that an oscillating signal appears predominantly in only one of the six combinations; the secondary signals appearing in any one of the remaining combinations are $<0.2$ of the prominent signal.

These results can be expressed more formally: the eigenfunctions are global and, to an accuracy of better than $20 \%$, are either symmetric or antisymmetric for reflection on the solar disk through the Sun's center $(\theta \rightarrow \pi-\theta, \phi \rightarrow \phi+\pi)$ and for reflection about the solar equator where $\theta$ and $\phi$ are defined in Figure 2. So for each observed oscillation, there exists at this time a set of symmetry properties.

These symmetry properties show that the Sun's observed axis of surface rotation is strongly reflected in the observed eigenfunctions. It is well-known that the spherical harmonic $Y_{l}^{m}(\theta, \phi)$ for the coordinate system defined in Figure 2 is also either symmetric or antisymmetric, depending upon the values of $l$ and $m$ for the two types of reflections discussed above. Therefore, these observations support the description of the horizontal spatial properties of the eigenfunctions by $Y_{l}^{m}$, and the $l$ and $m$ value for each oscillation can be classified as either even or odd.

Claverie et al. (1981) recently reported detecting a series of oscillating modes that they classified as high order with $l=1, m=0$. Whole disk velocity measurements such as these should have very low sensitivity to states of odd $l+m$ if the $(\theta, \phi)$ dependence of the eigenfunction is given by $Y_{l}^{m}$. Therefore, they should not see the $l=1, m=0$ or the $l=2, m= \pm 1$ states. However, they obtain strong signals for all three cases.

One way out of this dilemma is to propose a modification of the Sun that would remove the previously expected symmetry. Isaak (1982) has made such a proposal, suggesting that the Sun is an oblique magnetic rotator. But the symmetry property that this model removes, i.e., the existence of antisymmetric wavefunctions for reflection about the equator, is in fact one of the properties found in the observations reported here. Thus, there does appear to be a fundamental discrepancy between the two sets of observations. The results reported here indicate that solar rotation is the primary spherical symmetry-breaking phenomenon on the Sun.

As mentioned in the introductory remarks of this section, the observed symmetry properties may be quite restrictive in the search for the origin of the oscillations. First, let us consider the symmetry property for reflection through the center of the solar disk. 
The observed existence of a strong correlation between the motions of the limbs over a distance of 2000 arc sec clearly shows that the oscillations are spatially global, a result consistent with that found in Section 3. Second, consider the fact that the observed oscillations have symmetry properties that track the solar axis of rotation. These properties plainly point to the Sun as the origin of the observed oscillations and, in particular, rule out differential refraction effects in the Earth's atmosphere.

\section{Reproducibility of Power Density Spectra}

Inspection of Figure 3 shows that the spectrum is quite dense, a characteristic which is typical of the entire band from $125 \mu \mathrm{Hz}$ to $\approx 1.5 \mathrm{mHz}$. This is encouraging with respect to future work on the inverse problem, i.e., determining the internal structure of the Sun from the eigenfrequency spectrum. However, this complexity makes it quite difficult to make any meaningful comparison with results previously reported from SCLERA. In all of these preceding works, the frequency resolution that was used in the analysis was $\approx 30 \mu \mathrm{Hz}$. The peaks in these earlier spectra probably represented the superposition of several of the peaks in the work reported here.

However, the data set from the 1979 observations, in which the peaks are probably associated with individual eigenstates, can be divided into two independent subsets and the reproducibility of peaks in the power density spectra verified. This procedure employed the power density spectra used to determine the mean of the temporally noncoherent power level, i.e., the 18-day data set was divided into even- and oddnumbered subsets and their power density spectra intercompared. In this manner, two independent data sets were generated, both with a frequency resolution of approximately $0.3 \mu \mathrm{Hz}$. In the frequency range from 400 to $500 \mu \mathrm{Hz}$ and counting all peaks with a power density $>1.25 \times 10^{-3}$ (milli arc sec) $)^{2}$, there are 206 peaks in one spectrum and 230 in the second. Superimposing these two spectra, there are 175 peaks which coincide, to within $0.3 \mu \mathrm{Hz}$, from one spectrum to the other. No attempt has been made to remove the sidebands, i.e., the extra peaks, associated with an oscillation and introduced by the window function. However, a preliminary analysis indicates that about $\frac{2}{3}$ of the peaks counted in either power spectrum are sidebands. Taking this into account, one finds that there are approximately 70 independent peaks in the range from 400 to $500 \mu \mathrm{Hz}$ and that $80 \%$ of these are coincident.

This observed coincidence rate is eleven standard deviations away from that expected on the hypothesis that the peaks result from noise. However, this result should come as no surprise since it was found that $98.9 \%$ of the power density in the spectrum had coherence times $>2$ days.

\section{Amplitudes of Resolved Modes of Oscillation}

The mean power of the observed oscillatory signals in the 1979 observations is $2.5 \times 10^{-2}$ (milli arc sec $)^{2} / 0.28 \mu \mathrm{Hz}$ at $450 \mu \mathrm{Hz}$. The root mean square amplitude, $\left\langle a^{2}\right\rangle^{1 / 2}$, can be obtained by integrating the power density function of a single state with 
respect to frequency. This yields a mean square amplitude of

$$
\left\langle a^{2}\right\rangle^{1 / 2}=0.4 \mathrm{milli} \text { arc sec/state } .
$$

This will be a very useful number in future work on these oscillations.

A comparison of the power density observed here with that of Brown et al. (1978) is of interest. To do this, several factors must be considered: the difference in sensitivity of the two measurements ( $S_{i}$ vs $r$ ); the reporting of diameter vs radius amplitude; different sensitivities to spatially noncoherent signals; and different frequency resolution. Taking these factors into account and converting to a representation that permits direct comparison of these results with those of Brown et al., the power density of $2.5 \times 10^{-2}$ (milli arc sec $)^{2} / 0.28 \mu \mathrm{Hz}$ is equivalent to a power density of

$$
24(\text { milli arc sec) })^{2} / 30 \mu \mathrm{Hz}
$$

at $450 \mu \mathrm{Hz}$. This is to be compared to 28 (milli arc sec) $)^{2} / 30 \mu \mathrm{Hz}$, the value actually obtained by Brown et al. (1978).

\section{Atmospheric Differential Refraction and Seeing Effects Reduced to the Submilliarcsecond Level}

The mean power of the observed oscillating signals in the 1979 data is $2.5 \times 10^{-2}$ (milli arc sec) $)^{2} / 0.28 \mu \mathrm{Hz}$. As noted in Section 7 , this value compares quite well with the previously reported power density of Brown et al. (1978). Clearly, atmospheric differential refraction and seeing effects must be below this level if they are not to present any problem.

In the 1979 observations, large-scale differential refraction and seeing effects are estimated to be $\leqslant 3 \times 10^{-6}(\mathrm{~m} \text { arc sec })^{2} / 0.28 \mu \mathrm{Hz}$ and $\leqslant 1 \times 10^{-5}(\mathrm{~m} \text { arc sec})^{2} / 0.28$ $\mu \mathrm{Hz}$, respectively. These upper limits are considerably less than the observed oscillatory power given above. These low power levels for atmospheric effects were achieved as the result of two features of the observational method pertaining to the technique used to detect motion of the solar limb and to the number of positions on the solar limb which were examined. These features are discussed in this section.

As previously described, the oscillations were detected as changes in the location of the solar limb as defined by the application of the FFTD using a 6.8-arc sec scan amplitude relative to that defined by the FFTD using a 27.2-arc sec scan amplitude. This procedure reduces the amplitude of differential refraction effects by a factor of 300 or the corresponding power density by a factor of $300^{2}$ relative to those found in simple diameter measurements.

The manner in which this reduction comes about can be easily seen. The magnitude of the correlation, $C$, between column density fluctuations in the Earth's atmosphere measured along the two lines of sight to opposite solar limbs was found by Fossat et al. (1981) to be very close to 1 , i.e.,

$$
1-C=0.5 \times 10^{-2} \text {. }
$$


This measurement puts constraints on the characteristic horizontal scale of fluctuations in the atmospheric column density. In particular, this length in angular measure must be greater than the diameter of the Sun, a point also noted by Fossat et al. (1981). Therefore, to a good approximation, a Taylor series expansion can be made of the atmospheric refraction $R$ about the center of the Sun in the direction of the diameter being measured. Keeping terms to second order, we have

$$
R(r)=R(0)+R^{\prime} r+\frac{1}{2} R^{\prime \prime} r^{2}
$$

or

where

$$
\Delta R(r)=R^{\prime} r+\frac{1}{2} R^{\prime \prime} r^{2},
$$

$$
\Delta R(r)=R(r)-R(0) .
$$

Thus, in the combination of $S_{i}$, i.e.

$$
\left(\sum_{i=1}^{6} S_{i}\right) / 6
$$

used in Figure 3, the residual effect, $\Delta \boldsymbol{R}_{\Sigma S_{i}}$, is

$$
\Delta R_{\Sigma S_{i}}=2 R^{\prime}\left(r_{2}-r_{1}\right) \text {, }
$$

where $r_{1}$ and $r_{2}$ correspond to the radii defined by the FFTD 6.8- and 27.2-arc sec scan amplitudes, respectively. Note that second order effects drop out. For a diameter measurement defined by the FFTD 27.2-arc sec scan alone, the refraction effect, $\Delta R_{d}$, is

$$
\Delta R_{d}=2 R^{\prime} r_{2} .
$$

Thus we have

$$
\frac{\Delta R_{\Sigma S_{i}}}{\Delta R_{d}}=\frac{r_{2}-r_{1}}{r_{1}}
$$

The difference $r_{1}-r_{2}$ is $3.5^{\prime \prime}$ (see Section 2) so that

$$
\frac{\Delta R_{\Sigma S_{i}}}{\Delta R_{d}}=-3.5 \times 10^{-3}
$$

Thus, power density for large-scale differential refraction effects in the 1979 observations was reduced by a factor of $10^{5}$ below that present in the earlier diameter measurements.

Previous attempts, such as the work of Fossat et al. (1981), to infer the size of atmospheric differential refraction assumed that the correlation $C$ is very close to one, as supported by their observations, and considered only large-scale effects. Since large-scale differential refraction effects have clearly been reduced to a negligible level, differential refraction with horizontal length $\lambda_{a}<2 R$ may also receive attention. In the range $\lambda_{a}>100$ arc sec, the sensitivity to this noise source is also reduced by a factor 
similar to that given by Equation (19). The factor in this case is

$$
\frac{\Delta R_{\Sigma} S_{i}}{\Delta R_{d}}=\frac{4 \pi\left(r_{2}-r_{1}\right)}{\lambda_{a}}
$$

For $\lambda_{a}<100$ arc sec and a coherence length also $<100$ arc sec, the resulting power density is additionally reduced by a factor of 6 because the signal from six limb positions is averaged together. Thus the average power density from atmospheric differential refraction in the 1979 data has been reduced by a rather large factor over most of the spatial frequency range.

Atmospheric seeing is at the low level of $<1 \times 10^{-5}$ (milli $\left.\operatorname{arc~sec}\right)^{2} / 0.28 \mu \mathrm{Hz}$ because of the utilization of the FFTD in defining the position of the solar limb. The low sensitivity of the FFTD to seeing is one of its strong features (see Hill et al., 1975). The net seeing effect in these observations is also reduced by a factor of 3 below that present in the earlier diameter measurements because the data contain the average of the signals from six limb positions rather than only two.

Should any questions remain regarding the elimination of the Earth's atmospheric effects in the analysis of the 1979 observations, there is yet another consideration. The spatial symmetry properties of the observed signals as classified in Section 5 are indeed difficult to understand if the oscillations are the result of atmospheric effects. These symmetry properties comprise one of the five sufficient condition tests discussed by Hill et al. (1983). The symmetry of the signals for reflection about the center of the Sun establishes the global spatial character of the phenomenon. The contribution to a global signal from atmospheric seeing with an aplanatic patch size of the order of 10 arc sec must be reduced by many orders of magnitude below the seeing contribution to noise in the location of a single limb of $1 \times 10^{-5}(\mathrm{milli} \operatorname{arc~sec})^{2} / 0.28 \mu \mathrm{Hz}$ given above. Actually, for reflections through the center of the Sun, the oscillating signals were observed to be either symmetric or antisymmetric with the magnitude of the amplitudes of the two sides being equal to $\pm 20 \%$. Such properties would certainly make difficult an interpretation of the oscillations in terms of atmospheric differential refraction.

Finally, some additional statements can be made which are quite relevant. In Section 3, it was determined that the power of the temporally noncoherent signals was $1.1 \%$ of the mean peak power of the coherent component. Atmospheric effects which are temporally noncoherent must be less than or equal to this upper limit. Similarly, the spatially noncoherent atmospheric component must be less than $2 \pm 5 \%$ of the mean peak power. Diurnal terms, a potentially serious source of coherent signal arising from the atmosphere, are also not present, as can be seen by examining Figure 3.

Atmospheric effects have received much attention in work on solar diameter measurements. However, the analysis of the 1979 observations shows that these effects have been reduced to a nonsignificant level while not seriously degrading the sensitivity of the observations to the stable, oscillatory phenomena which are of interest. 


\section{Discussion}

This section brings together evidence for the interpretation of the individual spectral peaks as the resolved frequencies of individual normal modes of solar oscillation. Observational results are presented which are sufficient to demonstrate: (1) that the oscillatory phenomena are spatially global; (2) that the frequencies of the oscillations are stable to better than one part in 1000 over a period of 41 days; and (3) that the horizontal spatial characteristics of the oscillations have symmetry properties that identify an axis in the Sun coinciding with the solar rotational axis. Further, the observational program has been designed to reduce the effects of the Earth's atmosphere to a level well below the observed power density levels of the oscillations.

In order to develop these points, let us examine the postulate that the observed structure in Figure 3 is the result of noise. In this examination, the effects of the Earth's atmosphere can be quickly dismissed because of the extremely low sensitivity of the present observations to atmospheric effects, a feature built into the program (see Section 8). The oscillatory signals might also be arising within the observing instrument. However, this is not a viable explanation in light of the horizontal symmetry properties of the observed signals. The oscillations can be classified as either symmetric or antisymmetric for reflection on the solar disk through the center of the Sun and for reflection about the solar equator, properties which cannot be easily ascribed to instrumental effects but which are natural if the oscillations are solar in origin.

The noise postulate is thus reduced to a statement that the observed structure in Figure 3 is the result of noise, i.e., fluctuations, in the solar limb darkening function. Presumably, the use of the term noise in this case must be used to signify a local perturbation. It is quite clear that this is not the proper interpretation, as the oscillations are observed simultaneously at diametrically opposite limbs of the Sun with the magnitude of these diametrically derived signals equal to $\pm 20 \%$ and with the signals either in phase or out of phase with each other (see Section 5). These results are a direct demonstration of the global properties of the oscillations.

The interpretation of the signal as noise in lieu of global oscillations also presumbly implies certain temporal properties. That is, a noise source should not lead to oscillating features which are very stable in time. But only $1.1 \%$ of the observed signal was found to be temporally noncoherent for coherence times $>2$ days and a significant number of the oscillations was found to have coherence times $>41$ days (see Sections 3 and 4).

Thus, the postulate that the observed structure in Figure 3 is the result of noise fails on all accounts: the observed spatial and temporal properties are only consistent with the postulate that individual normal modes of oscillation of the Sun have been detected.

\section{Summary}

Evidence has been given which supports the conclusion that individual eigenstates of the Sun have been detected in the period range from $\approx 2 \mathrm{hr}$ to $\approx 10 \mathrm{~min}$. If the Sun's interior is represented fairly well by one of the standard solar models, then we have 
clearly observed both individual acoustic and gravity mode eigenstates of the Sun. The implications of this are obvious. We are at the threshold of an exciting era in the field of helioseismology in which it will be possible to probe the solar interior at an extremely quantitative level. If, on the other hand, individual gravity mode eigenstates have not been detected, the state of our knowledge of the solar interior is not as advanced as is generally believed, a prospect which is equally exciting.

\section{Acknowledgements}

The authors wish to acknowledge helpful discussions with Thomas Caudell and critical reading of the manuscript by Caudell and Philip Goode. This work was partially supported by the Astronomy Division of the National Science Foundation and the Air Force Office of Scientific Research.

\section{References}

Brown, T. M., Stebbins, R. T., and Hill, H. A.: 1978, Astrophys. J. 223, 324.

Caudell, T. P. and Hill, H. A.: 1980, Monthly Notices Roy. Astron. Soc. 193, 381.

Caudell, T. P., Knapp, J., Hill, H. A., and Logan, J. D.: 1980, in H. A. Hill and W. A. Dziembowski (eds.), Nonradial and Nonlinear Stellar Pulsation, Lecture Notes in Physics, No. 125, Springer-Verlag, Berlin, p. 206.

Claverie, A., Isaak, G. R., McLeod, C. P., van der Raay, H. B., and Roca Cortes, T.: 1981, Nature $293,443$.

Clayton, P. D.: 1973, 'A Precise Measurement of the Sun's Visual Oblateness', University of Arizona (Ph. D. Thesis).

Dicke, R. H.: 1978, in G. Friedlander (ed.), Proceedings Informal Conference on Status and Future of Solar Neutrino Research, BNL Report 50879, 2, Brookhaven National Laboratories, Upton, New York, p. 109.

Fossat, E., Grec, G., and Harvey, J. W.: 1981, Astron. Astrophys. 94, 95.

Gough, D. O.: 1978, Proceedings 2nd European Solar Meeting, Toulouse, France, March 8-10.

Groth, E. J.: 1975, Astrophys. J. Suppl. 29, 286.

Hill, H. A.: 1978, in J. A. Eddy (ed.), The New Solar Physics, Westview Press, Boulder, Colorado, Chapter 5.

Hill, H. A. and Caudell, T. P.: 1979, Monthly Notices Roy. Astron. Soc. 186, 327.

Hill, H. A. and Stebbins, R. T.: 1975, Astrophys. J. 200, 471.

Hill, H. A., Stebbins, R. T., and Oleson, J. R.: 1975, Astrophys. J. 200, 484.

Hill, H. A., Bos, R. J., and Caudell, T. P.: 1983, Solar Phys. 82, 129 (this volume).

Isaak, G. R.: 1982, Nature 296, 130.

Knapp, J., Hill, H. A., and Caudell, T. P.: 1980, in H. A. Hill and W. A. Dziembowski (eds.), Nonradial and Nonlinear Stellar Pulsation, Lecture Notes in Physics, No. 125, Springer-Verlag, Berlin, p. 394.

Oleson, J. R., Zanoni, C. A., Hill, H. A., Healy, A. W., Clayton, P. D., and Patz, D. L.: 1974, Appl. Opt. 13, 206.

Patz, D. L.: 1975, 'An Experimental Method to Determine Small Differences Between the Polar and Equatorial Solar Limb Profiles', University of Arizona (Ph. D. thesis).

Stratonovich, R. L.: 1963, Topics in the Theory of Random Noise, transl. from the Russian by Richard A. Silverman; Vol. 3 in the series: Mathematics and Its Applications, Gordon and Breach Science Publishers, Inc., New York. 